\title{
A representative interactive linear eddy model (RILEM) for non-premixed combustion
}

Author, co-author (Do NOT enter this information. It will be pulled from participant tab in

MyTechZone)

Affiliation (Do NOT enter this information. It will be pulled from participant tab in MyTechZone) 


\section{Abstract}

To further improve the efficiency and emissions profiles of internal combustion engines, many new combustion concepts are currently being investigated. Examples include homogeneous charge compression ignition ( $\mathrm{HCCl}$ ), stratified charge compression ignition (SCCl), lean stratified premixed combustion, and the use of high levels of exhaust gas recirculation (EGR) in diesel engines. The typical combustion temperatures in all of these concepts are lower than those in traditional spark ignition or diesel engines.

Most of the combustion models that are currently used in computational fluid dynamics (CFD) simulations were developed to describe either premixed or non-premixed combustion under the assumption of fast chemistry. The refinement of existing combustion concepts for highly efficient clean engines and the development of new ones would be greatly facilitated by the introduction of new computational tools and combustion models that are mode- and regimeindependent, i.e. capable of modeling both premixed and nonpremixed and also fast and non-fast chemistry. Such tools should enable more accurate simulation of combustion under non-standard conditions such as those established during low temperature combustion.

This paper presents a new regime-independent combustion modeling strategy for non-premixed combustion in which the linear eddy model (LEM) is used as a representative interactive regime-independent turbulent combustion model and coupled to a 3D CFD solver. Parameters and boundary conditions that determine the evolution of the LEM are supplied by the 3D CFD calculation and updated at each time step. The LEM is then solved for the corresponding time step, providing the $3 D$ CFD code with an updated composition state.

This new representative interactive linear eddy model (RILEM) is used to simulate an n-heptane spray, demonstrating some potential to describe spray combustion processes.

\section{Introduction}

Many of the combustion models in use today work reasonably well for traditional diesel (non-premixed) or gasoline (premixed) engines. They take advantage of the physical characteristics of different combustion modes (premixed or non-premixed) and are often based on the assumption of fast chemistry, which creates a clear separation between the turbulent and chemical time and length scales. As a result, they are usually not applicable to the modeling of combustion modes other than those for which they were designed, and their usefulness as

2

Page 2 of 12 predictive tools for the development of new engine concepts may be limited. Among the most widely used models are flamelet models for premixed and non-premixed combustion, which rely on an assumption of fast chemistry that implies the formation of laminar flame structures embedded in a turbulent flow field. The coupling between turbulence and chemistry in flamelet-type models is usually achieved in a parametric way (e.g. via the scalar dissipation rate in non-premixed combustion or by means of turbulent velocity fluctuations in premixed combustion), which means that there is no direct interaction between chemistry, molecular transport, and turbulence. However, if the chemical time scales are not fast compared to the fastest turbulent time scales (as is the case during low temperature combustion, ignition, and re-ignition), it becomes essential to accurately describe the interactions between chemistry, molecular transport, and turbulence in order to obtain realistic results. Other popular classes of combustion model for engine applications involving non-premixed combustion are stirred/partially stirred reactor models and the volume reactor fraction model (VRFM) [3], in which the chemistry is directly integrated. However, these models do not provide a characteristic length or velocity scale of combustion and therefore cannot predict/model flame structure. Ideally, a predictive combustion model that can handle multi-mode and multi-regime combustion conditions would [2]:

- describe the interactions between turbulence, chemistry, and molecular transport in a direct (nonparameterized) way,

- feature a closed-form treatment of chemistry and molecular transport,

- preserve structures (flames) that depend on the coupling of reaction and diffusion,

- enable resolution at all length and time scales, and

- make no assumptions about statistical distributions (i.e. not rely on presumed PDFs).

Existing regime- and mode- independent combustion models include transported PDF models with structure-based mixing models [16] and low-dimensional stochastic models such as LES-LEM, in which the linear eddy model of Kerstein [10] is used as a sub-grid model in a large-eddy simulation (LES) [8, $9,21,22]$. In LES-LEM a one-dimensional representation of the turbulent combustion process is solved in each LES cell by resolving all spatial and temporal scales, as is done in direct numerical simulations. LEM is distinguished from most other modeling strategies by its use of this fully resolved onedimensional representation that does not involve sub-grid scale modeling. Because it describes direct interactions between turbulent mixing, diffusion and chemical reactions, the model is capable of predicting highly unsteady effects such as extinction and re-ignition without requiring any modification. One 
disadvantage of the LES-LEM approach is its high computational cost: the resolution of all temporal and spatial scales within the one-dimensional LEM domain is very timeconsuming. Large-scale applications therefore require a wellparallelized environment together with the adoption of chemistry acceleration techniques such as in situ adaptive tabulation (ISAT) or neural network strategies. Here we present a LEM approach in which we solve only a few representative linear-eddy model instantiations in the computational domain. The goal was to create a modeling approach that retains the key advantages of a full LES-LEM, namely regime and mode independence, at acceptable computational costs. Our approach does not exhibit all of the properties identified above as being desirable for mode- and regime-independent modeling because it uses a presumed PDF approach and a globally representative model rather than one that is locally regime-independent. The approach has some similarities with the representative interactive flamelet (RIF) approach of Pitsch et al. [19] but features some distinct advantages such as regime independence and intrinsically variable scalar dissipation rates.

The linear-eddy model has been used both as a stand-alone tool and an LES sub-grid model for describing scalar mixing and turbulent combustion under various flow and combustion conditions [18, 20, 21, 22, 23]. The literature features only a few publications reporting the use of LEM as a sub-grid model in the context of reciprocating engines. The first of these was presented by Some and Menon [23], who implemented LESLEM in KIVA and compared its performance in modeling fuelair mixing in a direct injection spark ignition (DISI) engine to that achieved using KIVA-RANS and standard KIVA-LEM. Combustion and heat release were not considered. As expected, the LEM simulations provided a much better resolution of turbulent flow structures than the RANS simulation. However, no comparisons with experimental data were provided. Steeper et al. [24] used LEM as a sub-grid combustion model within a URANS approach to investigate the influence of various generic initial fuel and temperature stratifications on pressure histories in an automotive $\mathrm{HCCl}$ engine. Using the coupled URANS-LEM approach, they were able to qualitatively describe the interaction of the spatial fuel distribution, turbulent mixing, and combustion. Their simulations used only one LEM domain and a simple 2-step, 6species mechanism for $n$-heptane; consequently, their results were qualitative rather than reliably quantitative.

\section{Mathematical model}

\section{The Linear Eddy model}

The Linear-Eddy Model was proposed by Kerstein [10] as a scalar mixing model for non-reacting flows, and was subsequently extended to describe reactive flows. It has been discussed at length in the literature $[10,11,12,13,14]$ and is therefore only briefly summarized here. Essentially, it describes turbulent reactive flows in terms of two concurrent processes. The first of these processes describes the effects of dilatationinduced advection, molecular diffusion, and chemical reactions. It involves the time advancement of the reactive zero-Machnumber equations on a one-dimensional domain and enables resolution at all possible spatial and temporal scales. The second process, turbulent transport, is implemented as a stochastic sequence of statistically independent eddy events.

\section{LEM diffusion and chemical reactions}

The LEM code used in this study is based on a C++ implementation of the one-dimensional turbulence (ODT) model [1] of Lignell et al. [2]. It features adaptive grid refinement and a Lagrangian (fixed mass) formulation of the balance equations. The balance equations for species mass fractions $Y_{\mathrm{s}}$ and mass-specific mixture enthalpy $h$ are:

(1) $\rho \frac{\mathrm{D} Y_{s}}{\mathrm{D} t}=-\frac{\mathrm{d} j_{s}}{\mathrm{~d} x}+M_{S} \dot{\omega}_{s}$,

(2) $\rho \frac{\mathrm{D} h}{\mathrm{D} t}=\frac{\mathrm{d} p}{\mathrm{~d} t}-\frac{\mathrm{d} q}{\mathrm{~d} x}-\sum_{s} j_{s} \frac{\mathrm{d} h_{s}}{\mathrm{~d} x}-\sum_{s} h_{s} M_{s} \dot{\omega}$,

where $\rho$ is the density, $j_{s}$ the species diffusion flux, $M_{s}$ the species molar mass, $p$ the pressure, $q$ the heat flux, $h_{s}$ the enthalpy of species $s$ including the heat of formation, and $\dot{\omega}_{s}$ the chemical source term of species $s$. For the equation of state of a mixture of ideal gases we have $p=\rho T \sum_{s} Y_{S} R_{S}$, with $R_{s}$ denoting the individual gas constant of species $s$. The temperature is determined via the caloric equation of state, $h(T)=\Delta h_{s}^{0}+\int_{T^{0}}^{T} c_{p, s}(T) \mathrm{d} T$, where $\Delta h_{s}^{0}$ is the standard heat of formation of species $s$ and $c_{p, s}$ denotes the mass specific heat capacity at constant pressure. The global mass balance in a Lagrangian formulation is $\rho \Delta x=$ const.

The governing equations are solved using a second order accurate scheme with central discretization of the diffusion terms; Strang's operator splitting technique is used for integration in time between successive eddy events. The stiff chemical source term as well as all transport and 
thermodynamic variables are evaluated using the software package Cantera [5]. The stiff chemical source term is integrated using an implicit BDF method.

\section{LEM turbulent advection}

In the LEM concept, turbulent advection is implemented explicitly by stochastic eddy events. Each eddy event involves a rearrangement of all scalar quantities using so-called 'triplet maps' which mimic the influence of turbulent vortices. The effect of a triplet map is a threefold compression of the scalar fields in a selected spatial interval whose size is denoted by $I$. See Figure 1 for an illustration. This map increases the scalar gradients within the selected interval in a way that is analogous to the effect of compressive strain in turbulent flow, without creating discontinuities. Three quantities are needed to specify an eddy event: the eddy size $I$, the eddy's location $x$ within the domain, and the time of the eddy event.

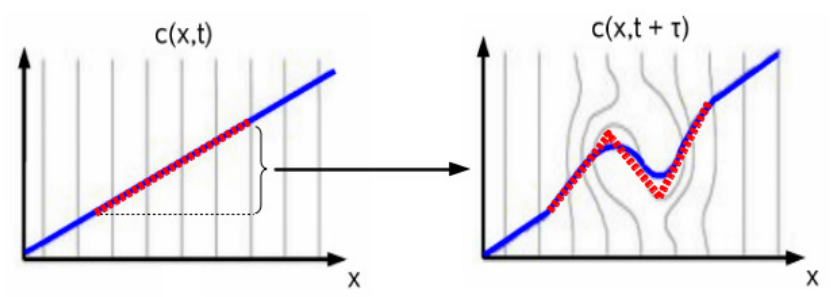

Figure 1: Qualitative picture of a triplet map in LEM (red symbols) superimposed on a representation of the analogous two-dimensional (2D) eddy motion (gray) and its effect on a 1D property profile (blue). Left: before mapping, right: after mapping.

In LEM, the eddy size is sampled from a prescribed size distribution. Assuming Kolmogorov inertial-range scaling, the eddy size distribution is given by [11]

(3) $f(l)=\frac{5}{3} \frac{l^{\frac{-8}{3}}}{\eta^{\frac{-5}{3}}-l_{t}^{\frac{-5}{3}}}$.

Using the turbulent Reynolds number

(4) $R e_{t}=\frac{u^{\prime} l_{t}}{v}$

where $v$ is the kinematic viscosity, and $\mathrm{u}^{\prime}, \mathrm{I}_{\mathrm{t}}$ are the integral velocity and length scale, respectively, and the Kolmogorov scale $\eta$ is determined from the inertial scaling law $\eta=$ 4

Page 4 of 12
$N_{\eta} l_{t} R e_{t}^{-3 / 4}$. Here $N_{\eta}$ is an empirical constant. The eddy event frequency per unit domain length is determined by [11]

(5) $\lambda=\frac{54}{5} \frac{v R e_{t}}{C_{\lambda} l_{t}^{3}} \frac{\left(\frac{l_{t}}{\eta}\right)^{\frac{5}{3}}-1}{1-\left(\frac{\eta}{l_{t}}\right)^{\frac{4}{3}}}$

where $\mathrm{C}_{\lambda}=15$ and $\mathrm{N}_{\eta}=10.76$ are model constants with values taken from the literature [4]. The LEM parameterization corresponds to a turbulent diffusivity of $D_{t}=u^{\prime} l_{t} / C_{\lambda}$ which can be related to the scalar dissipation rate via $X^{C F D}=2^{*} D_{t} / l_{t}^{2} . I_{t}$, $R e_{t}, D_{t}$, and the fluid property $v$ are the defining parameters of the linear-eddy model. In coupled CFD-LEM simulations they are passed as time-dependent parameters from the CFD side to the LEM. In the representative model described below we have one LEM instance and the LEM parameters must be chosen to be representative for the whole CFD domain. Conversely, in fully coupled LES-LEM simulations with LEM instances in each CFD cell, these parameters are local and therefore enable locally regime- and mode-independent modeling of turbulent combustion.

The eddy location is randomly sampled from a uniform distribution of eddies over the domain and the eddy time is sampled under the assumption of a Poisson process with a mean eddy occurrence time $\Delta t_{e d d y}=(\lambda L)^{-1}$, where $L$ is the domain size.

\section{RILEM}

The RILEM model is based on a new modeling approach that uses the LEM as a subgrid combustion model in a representative way. It aims to retain the LEM's advantages of mode- and regime-independent combustion modeling at an affordable computational cost. In contrast to LES-LEM, a standard RANS approach is used for turbulence modeling.

As shown in Figure 2, the RILEM model consists of a CFD solver (OpenFOAM in this case) and a representative LEM model coupled to the CFD solver. In the current implementation, the LEM provides only mass fraction values to the CFD (see below).

On the CFD side the standard set of equations for global mass, momentum, and enthalpy are solved together with a standard Lagrangian spray model including single component fuel evaporation. Turbulence is modeled using the standard $K-\varepsilon$ model. To characterize turbulent fuel mixing, additional 
transport equations for the mixture fraction $\widetilde{\boldsymbol{Z}}$ and the variance of the mixture fraction $\widetilde{Z^{\prime \prime 2}}$ are solved:

(6) $\frac{\partial(\bar{\rho} \widetilde{Z})}{\partial t}+\nabla \cdot(\bar{\rho} \tilde{u} \tilde{Z})=\nabla\left[\frac{\mu}{s c} \nabla \tilde{Z}\right]+\dot{S}_{\text {evap }}$

(7) $\frac{\partial\left(\bar{\rho} \widetilde{Z^{\prime \prime 2}}\right)}{\partial t}+\nabla \cdot\left(\bar{\rho} \tilde{u} \widetilde{Z^{\prime \prime 2}}\right)=\nabla \cdot\left[\frac{\mu_{t}}{s c} \nabla \widetilde{Z^{\prime \prime 2}}\right]+\frac{2 \mu_{t}}{s c}(\nabla \tilde{Z})^{2}-\tilde{\chi}$

Here $\dot{S}_{\text {evap }}, \mu_{t}, S c$ are respectively the source term due to evaporation, the turbulent viscosity, and the turbulent Schmidt number (which takes a constant value of 0.7 ).

The scalar dissipation rate $\tilde{\chi}$ is modeled as

(8) $\tilde{\chi}=c_{\chi} \frac{\epsilon}{\mathrm{k}} \widetilde{Z^{\prime \prime 2}}$,

with $c_{\chi}=2$.

The energy budget in our formulation is solved in the form of an equation for the total enthalpy $\tilde{h}$ :

(9) $\frac{\partial(\bar{\rho} \widetilde{h})}{\partial t}+\nabla \cdot(\bar{\rho} \tilde{u} \tilde{h})=\frac{\mathrm{d} \tilde{p}}{\mathrm{~d} t}-\nabla \cdot j+\dot{q}_{\text {evap }}$

where $j$ is the heat flux vector and $\dot{q}_{\text {evap }}$ is the enthalpy source due to droplet evaporation, which is provided by the spray model. Viscous heating has been neglected here, which is a reasonable assumption for low Mach-number flow.

Once the enthalpy equation is solved the temperature can be calculated via the caloric equation of state:

(10) $\widetilde{h}=\sum_{s=1}^{N} \widetilde{Y_{S}} h_{s}(\widetilde{T})$

Here, $h_{s}$ denotes the mass-specific enthalpy of species $\mathrm{s}$ including the heat of formation and the temperature dependent sensible enthalpy. The Favre-averaged species mass fractions $\widetilde{Y_{S}}$ in each cell of the computational domain are obtained by integrating LEM mass fraction values mapped onto mixture fraction space using a presumed $\beta$-PDF for the mixture fraction:

(11) $\widetilde{Y}_{S}=\int_{0}^{1} P\left(Z ; \widetilde{Z}, \widetilde{Z^{\prime \prime 2}}\right) Y_{S}^{L E M}(Z) \mathrm{d} Z$

Here, $Y_{S}^{L E M}(Z)$ denotes the mass fraction of species $s$ obtained on the representative LEM, which has been mapped onto mixture fraction space. This mapping differs from that in flamelet models in an important way: due to the stochastic nature of the LEM, an arbitrary number of different thermodynamic states are possible for a given mixture fraction value. This variability of states for a fixed mixture fraction reflects the inherent variability of scalar dissipation rates in the LEM. The probability density function of the scalar dissipation rate is an outcome of the solution and not - as in flamelet models - an input.

The basic structure of the RILEM code is presented in figure 2. CFD and representative LEM solutions are time advanced in an alternating way. First the fluid dynamics are solved for one time step on the CFD side. Then the LEM is provided with updated variables for the pressure change, a characteristic turbulent length and velocity scale, and information about the evaporated fuel mass. The fuel is inserted in the middle of the LEM line, representing an unsteady fuel injection process. The following procedure was developed to model the spatial distribution of the fuel evaporation rates on the LEM line: We first sort the fuel evaporation rates $\dot{r}_{c}$ on the CFD side in descending order. Each rate is associated with a ratio of the CFD cell volume to the global volume of the CFD domain, $\alpha_{c}=V_{c} / V$. We then apply the fuel evaporation rates to the LEM domain in descending order, starting in the middle of the LEM domain such that each fuel evaporation rate precisely covers the associated fraction $\left(\alpha_{c}\right)$ of the LEM domain and is evenly distributed with respect to the domain's center, producing a symmetric fuel distribution. To avoid the introduction of numerical boundary layers, the LEM is time advanced using periodic boundary conditions. After fuel-vapor insertion, the LEM is time advanced to the same time point as the CFD code. The newly calculated species mass fractions obtained from the LEM are then mapped from 1D LEM space onto mixture fraction space by conventional averaging of each mass fraction over LEM cells with the appropriate mixture fraction. Having obtained species mass fractions over mixture fraction space and the presumed mixture fraction PDF, it is possible to determine the species distribution in every CFD cell for the next time step. Moreover, equation () can be used to calculate the temperature of each CFD cell in the next time step. Because each LEM cell hosts a complete thermodynamic state - including the temperature - we have two temperature values available, one on the CFD side and one on the LEM side, which might not match well. While this is a common occurrence in both flamelet and LES-LEM models, a large difference between the temperatures on the LEM and CFD sides may indicate that the LEM's results are not representative of the current combustion situation.

5

Page 5 of 12 


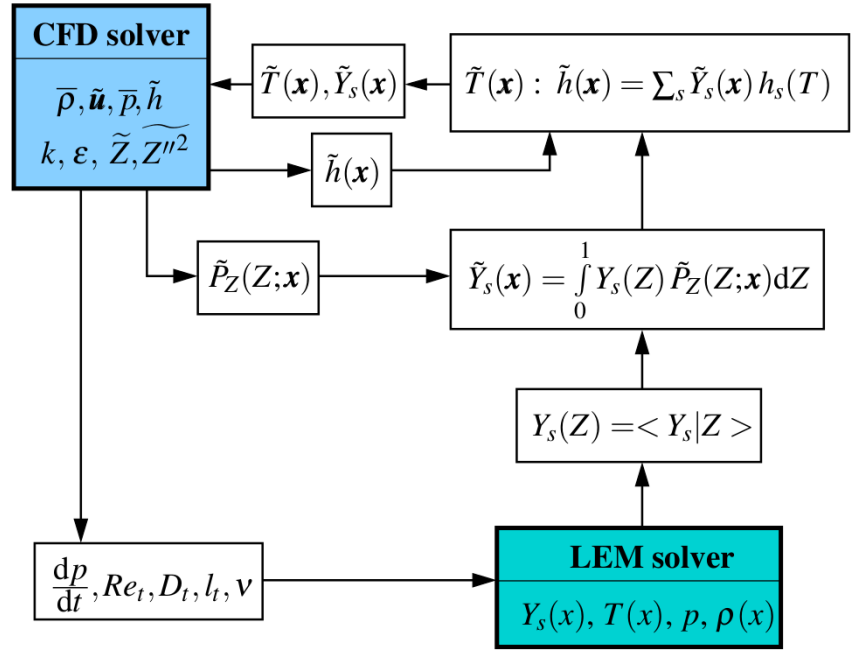

Figure 2: The code structure of the RILEM

\section{Chemical mechanism}

The chemical reaction mechanism for $n$-heptane combustion used in this work is the reduced mechanism of Maroteaux and Noel [6], which features 26 chemical reactions and 25 species. This mechanism can predict ignition delay times and heat release rates under conditions relevant to engine applications but does not account for the formation of pollutants.

\section{Results and discussion}

To demonstrate the new model's performance, it was used to perform numerical simulations of combustion in a high pressure and high temperature spray combustion chamber. The case examined has previously been investigated in a series of numerical simulations [25]. High pressure high temperature combustion chambers are alternatives to conventional combustion chambers and were developed to facilitate the study of sprays under engine-like conditions. Figure 3 shows the grid of the computational domain, which extends over a volume of $1 \mathrm{~cm} \times 1 \mathrm{~cm} \times 10 \mathrm{~cm}$. The nozzle is located in the middle of the chamber on the upper wall and has a diameter of $0.19 \mathrm{~mm}$. The chamber is fueled with an $\mathrm{n}$ heptane spray. All CFD simulations were performed using OpenFOAM 2.0.x with a $41 \times 41 \times 100$ grid containing 178164 gridpoints over the entire three-dimensional domain. An adiabatic wall boundary condition was imposed, with zero gradient conditions for all dependent variables. All simulations were conducted in serial mode on a $2.0 \mathrm{GHz}$ Intel Xeon CPU.

6

Page 6 of 12
The computation time was typically around 18 hours for a LEM of 4000 gridpoints (due to the adaptive mesh refinement in the LEM code, the number of gridpoints varies over time). The computational time on the LEM side is naturally very dependent on the grid size and the chosen chemical mechanism, and can therefore be reduced by scaling down the LEM line or using a simpler chemical mechanism. The boundary conditions were $800 \mathrm{~K}$ for the temperature of the air in the chamber and 42 bar pressure. The injected mass of fuel is $6 \mathrm{mg}$ and the (measured) rate profile is shown in in fig. 4 .

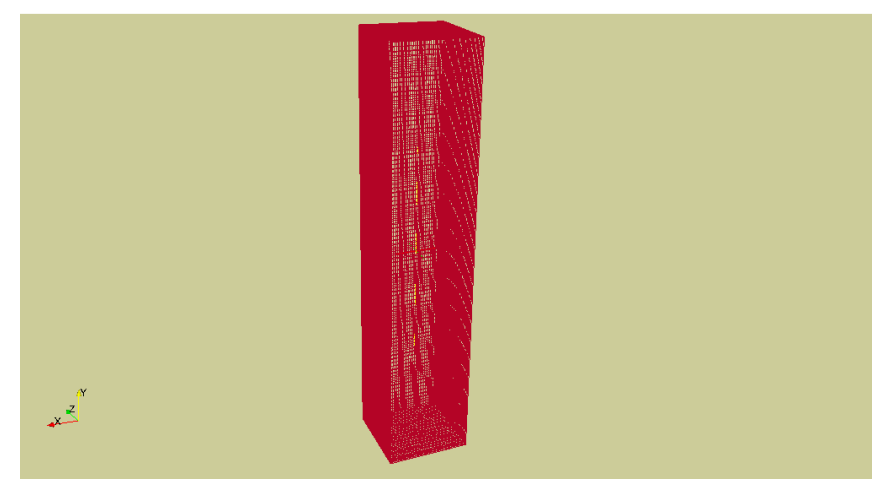

Figure 3: The numerical mesh used in the simulations

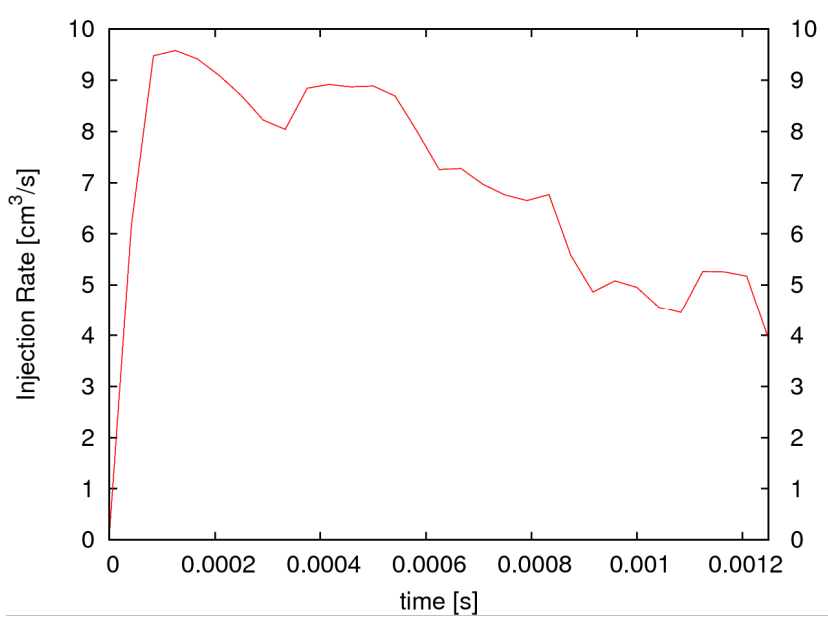

Figure 4: Fuel injection rate

\section{Comparison with Experiments}

The numerical predictions of combustion behavior obtained using RILEM depend strongly on the distribution of the mixture fraction $\tilde{Z}$ and the mixture fraction variance $\widetilde{Z^{\prime \prime}}$ because these variables define the mixture fraction PDF that is used to compute Favre-averaged mass fractions. The mapping of the representative LEM solution onto physical space in the CFD 
domain is completely and exclusively dependent on the mixture fraction PDF, as shown in Fig. 2. Both the mixture fraction and its variance depend on the rate of fuel evaporation, which is obtained from the spray model. A key variable to consider when assessing the performance of a spray model in describing the spray breakup process is the vapor penetration depth, which is defined as the distance between the nozzle and the most distant point at which the fuel vapor mass fraction is greater than five per cent. Figure 5 compares the vapor penetration depth calculated using the widely used KelvinHelmholtz Rayleigh-Taylor break model with default coefficients to the analytical solution reported by Wakuri et al. [26], who based their derivation on the conservation of momentum and assumed that the depth of penetration is proportional to $t^{1 / 2}$. The agreement between the calculated penetration depth and the analytical solution is quite reasonable in this case.

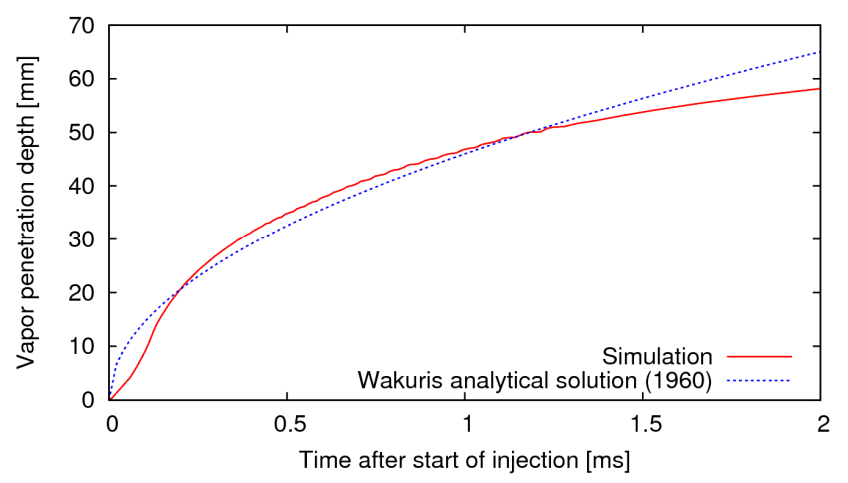

Figure 5:Vapour penetration depth

The predicted ignition delay time under the studied conditions under is about $0.8 \mathrm{~ms}$. For comparative purposes, shock tube and engine experiments conducted by Ciezki et al. [27] yielded ignition delays of around $0.7 \mathrm{~ms}$ and $1.0 \mathrm{~ms}$ for homogeneous mixtures and liquid sprays, respectively, at $800 \mathrm{~K}$ and 42 bar. In [25] Pitsch et al. calculated ignition delay times with a flamelet model of $1.5 \mathrm{~ms}$. The ignition delay times computed with RILEM here are a bit closer to the experimental data. In the future, the new model will be used to investigate the effects of mixture inhomogeneity on ignition delay.

To demonstrate the qualitative performance of the RILEM model, the temperature on the LEM line, the temperature of the CFD domain and some species mass fractions over mixture fraction space before and after combustion are shown in figures 7-15.

Fig. 6 and 7 show the temperature and mass fraction values on the LEM line at $t=0.5 \mathrm{~ms}$ where some fuel has been already injected. The LEM line was initialized with pure air at a temperature of $800 \mathrm{~K}$ and a pressure of 42 bar. As described above, fuel on the LEM line is inserted in the middle of the domain and is subsequently advected by turbulent eddies while diffusing towards the left and right ends of the domain over time. This is reflected in Fig. 6 where we see two temperature peaks of $T=970 \mathrm{~K}$ and $T=980 \mathrm{~K}$ at locations to the left and right of the domain center, where the mixture is almost stoichiometric. The central domain with the two temperature peaks is surrounded by air that remains in its initial state. The temperature of the two peaks corresponds to the low temperature ignition step in the two-step ignition process that occurs during $n$-heptane oxidation. At this stage of the simulation, the fuel has not ignited completely and the maximum temperatures observed are far below the adiabatic flame temperature achieved under stoichiometric conditions. In between the two temperature peaks we have inhomogeneous fuel-rich conditions with high local gradients between $x=0.04$ $\mathrm{mm}$ and $x=0.05 \mathrm{~mm}$ due to turbulent mixing that blends combustion products with fresh air. In this area we have elevated temperatures of approx. $870 \mathrm{~K}$, indicating the initiation of chemical reactions under fuel-rich conditions due to the mixing of fuel and air by turbulent eddies. To the right of this area, at $x \approx 0.06 \mathrm{~mm}$, the unburned state prevails. The asymmetry of the solution between the two temperature peaks due to mixture inhomogeneities arising from turbulent mixing may lead to localized enhancements or suppression of ignition; the ability to describe and resolve the structures of such inhomogeneities is a particularly useful characteristic of the unsteady LEM.

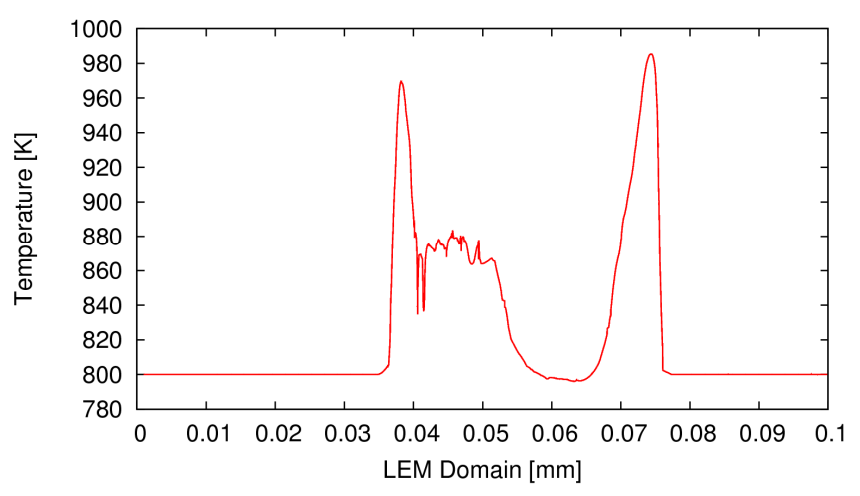

Figure 6: Temperature on the LEM line at $t=0.5 \mathrm{~ms}$

7

Page 7 of 12 


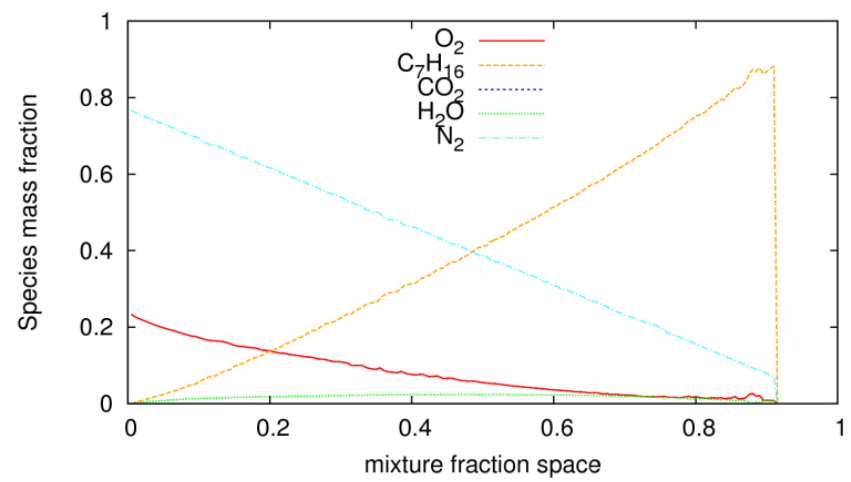

Figure 7: Mass fractions of individual chemical species within the mixture fraction space of the LEM at $t=0.5 \mathrm{~ms}$

Fig. 7 shows the mass fractions of selected major species $\left(\mathrm{C}_{7} \mathrm{H}_{16}, \mathrm{O}_{2}, \mathrm{CO}_{2}, \mathrm{H}_{2} \mathrm{O}\right.$, and $\left.\mathrm{N}_{2}\right)$ within mixture fraction space at $t$ $=0.5 \mathrm{~ms}$. The highest mixture fraction value is $Z \approx 0.9$ rather than 1 because it is limited by the amount of evaporated fuel. At this stage of the combustion process, the profiles are very similar to those obtained for the unburned state using flamelet models. At the upper end of the mixture fraction space, we observe some wiggles in the profiles due to turbulent mixing.

Figures 8 and 9 show the distributions of the mixture fraction and temperature, respectively, within the CFD domain at $T=$ $0.5 \mathrm{~ms}$. Figure 10 shows the distributions of both of these variables along the center line of the simulated chamber. The maximum mixture fraction value of $Z \approx 0.88$ is consistent with the results obtained on the LEM side, as shown in Figure 7. The temperature directly beneath the nozzle is below $800 \mathrm{~K}$ (as shown in Figures 9 and 10) due to fuel evaporation. Further away from the nozzle (at $x \approx 0.08 \mathrm{~m}$ in Fig. 10), low temperature kinetics have heated the mixture from its initial temperature of $800 \mathrm{~K}$ to approximately $1050 \mathrm{~K}$.

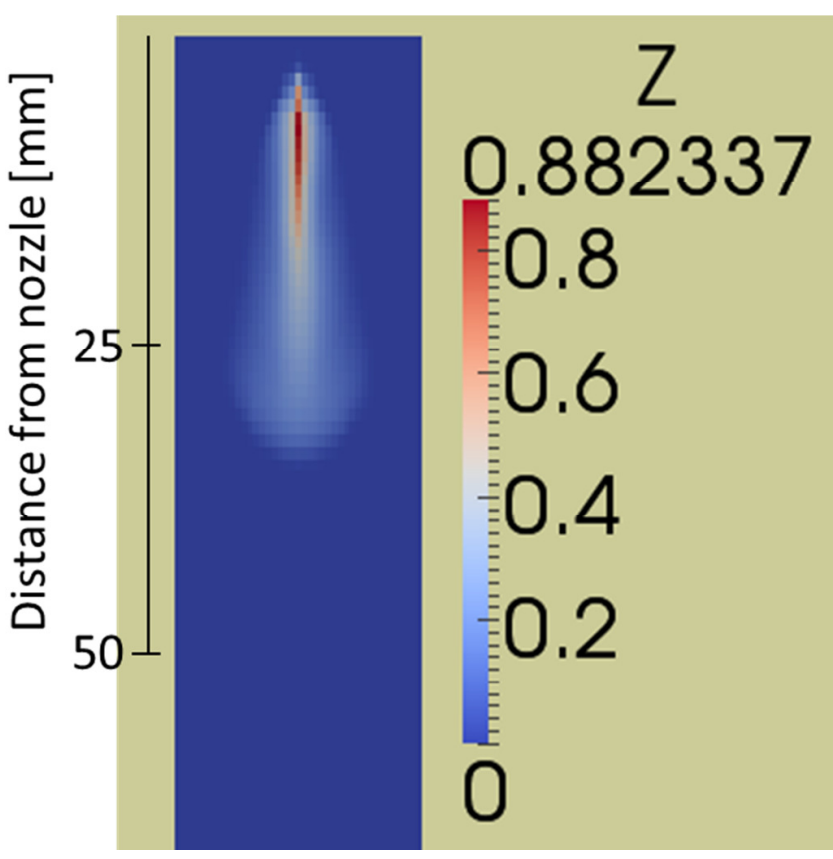

Figure 8: Mixture fraction distribution within the CFD domain at $t=0.5 \mathrm{~ms}$. The figure shows data for a plane extending through the middle of the chamber.

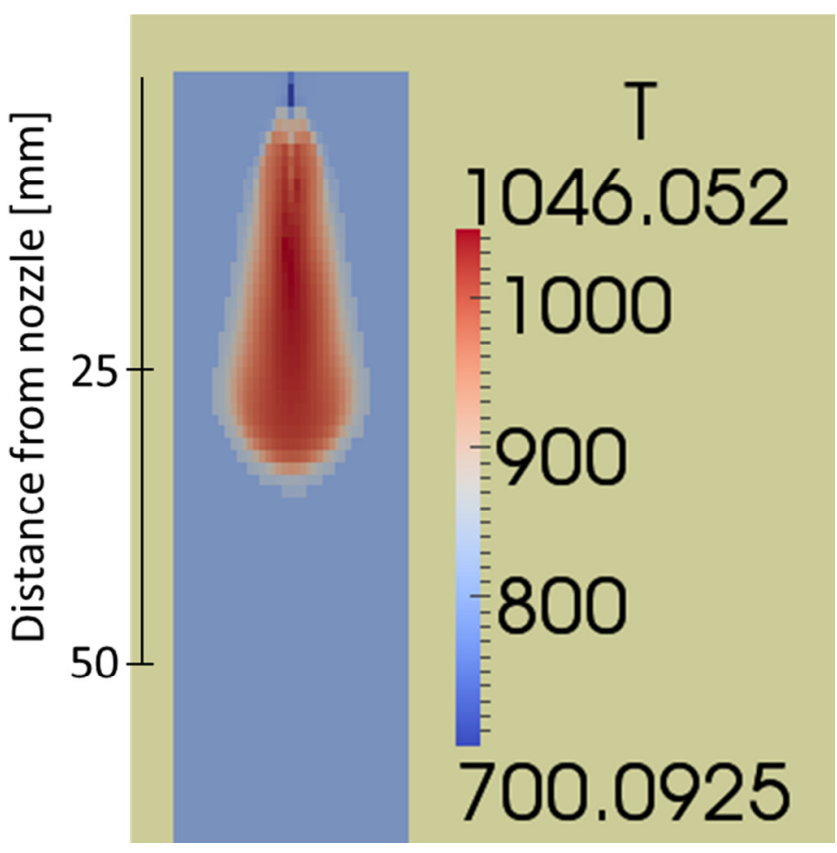

Figure 9: Temperature distribution within the CFD domain at $\mathrm{t}=$ $0.5 \mathrm{~ms}$. The figure shows data for a plane extending through 
the middle of the chamber.

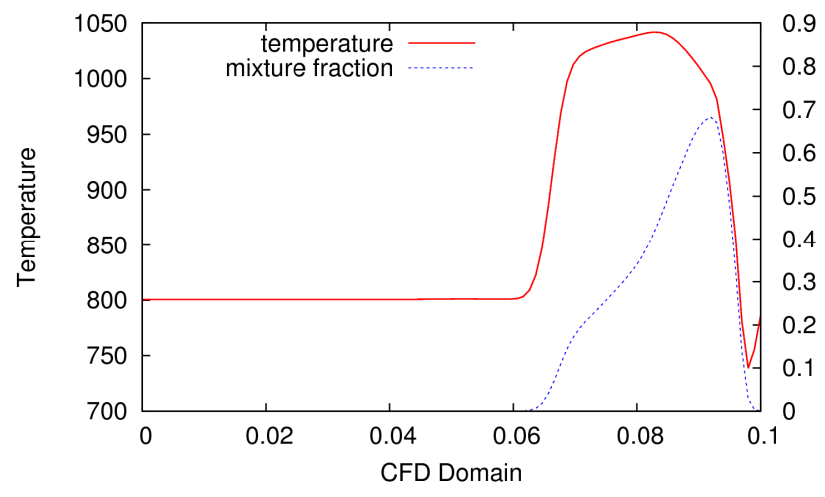

Figure 10: Interpolated values of the temperature and mixture fraction on a line running along the length of the simulated combustion chamber (in the y direction as defined in Figure 3) within the CFD domain at $t=0.5 \mathrm{~ms}$. On the horizontal axis of the plot, 0 corresponds to a position at the bottom of the chamber and a value of 0.1 corresponds to a position immediately below the nozzle.

Under the studied starting conditions, the predicted ignition delay time was around $0.8 \mathrm{~ms}$. Figures $11-15$ show results from the LEM and CFD domains after ignition at $t=1.1 \mathrm{~ms}$. Figure 11 shows the temperature on the LEM line, indicating that the flame temperature after ignition is around $2400 \mathrm{~K}$. The fuel is inserted in the middle of the LEM line, creating two regions with near-stoichiometric conditions, one on each side of the fuel-rich inner zone. These near-stoichiometric zones move towards the ends of the LEM domain over time. At the point in time captured in Figure 11, only one of the nearstoichiometric zones is burning due to turbulent motion on the LEM line, which can suppress mixture ignition.

Fig. 12 shows species mass fractions on the LEM side in relation to mixture fraction space at $t=1.1 \mathrm{~ms}$. The spike in the mass fraction of $\mathrm{CO}_{2}$ between $Z=0.066$ and $Z=0.1$ indicates that the mixture undergoes complete combustion under the indicated conditions, and corresponds to the temperature peak of $T \approx 2400 \mathrm{~K}$ at $Z \approx 0.08$ in Fig. 11. The zigzag profile observed for all species other than the inert $\mathrm{N}_{2}$ is caused by turbulent mixing on the LEM line and reflects the stochastic nature of the LEM. The species mass fractions shown here are a conventional ensemble average over the different states along an LEM line of constant mixture fraction. As discussed above, these different states with identical mixture fraction values can be interpreted as representations of fluctuations in the scalar dissipation rate within the LEM. Ideally, if the LEM domain were long enough we would expect smooth profiles for

9

Page 9 of 12 the averaged species over mixture fraction space. The zigzag profiles may thus indicate that we are not fully capturing the statistical fluctuations in the scalar dissipation rates. The statistical properties of the RILEM approach will be examined in future studies.

Figures 14 and 15 show the temperature distribution in the central plane of the CFD domain and along the symmetry axis at $\mathrm{t}=1.1 \mathrm{~ms}$. The temperature peaks at around $2100 \mathrm{~K}$, which is substantially lower than the maximum value of $2400 \mathrm{~K}$ observed along the LEM line (see Fig. 11). This is due to the absence of an enthalpy defect due to latent heat of the evaporating fuel, which is considered on the CFD side. The missing enthalpy defect in the LEM will be rectified in the next version of the RILEM model. The temperature peak occurs at around $Z=0.1$ (see Figs. 13 and 14), which is consistent with the report that flames start burning under slightly rich conditions [27].

Pitsch et al. [25] calculated the same case as the one presented in this paper with a RIF model. In [25] they show spatial temperature distributions along the axis for several time points from $2 \mathrm{~ms}$ to $4 \mathrm{~ms}$. The observed maximum temperatures of about $2250 \mathrm{~K}$ are comparable with the maximum temperature of $2200 \mathrm{~K}$ at the axis observed in Fig. 15 for the RILEM model.

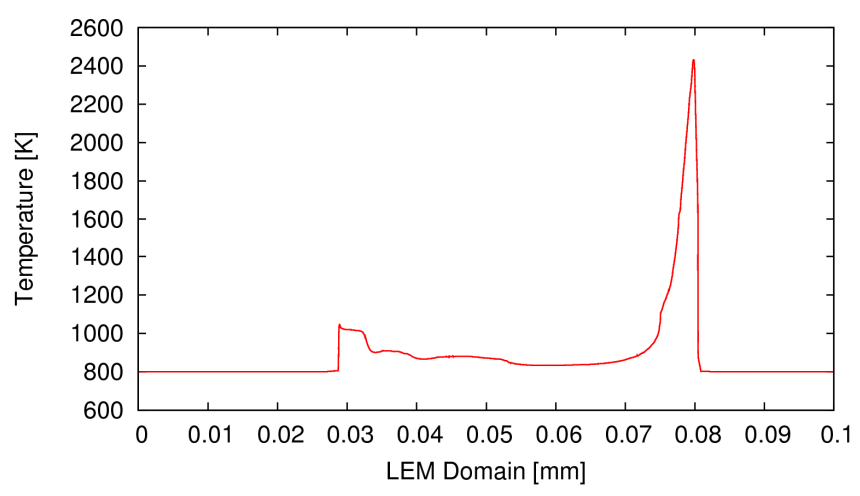

Figure 11: Temperature on the LEM line at $t=1.1 \mathrm{~ms}$ 


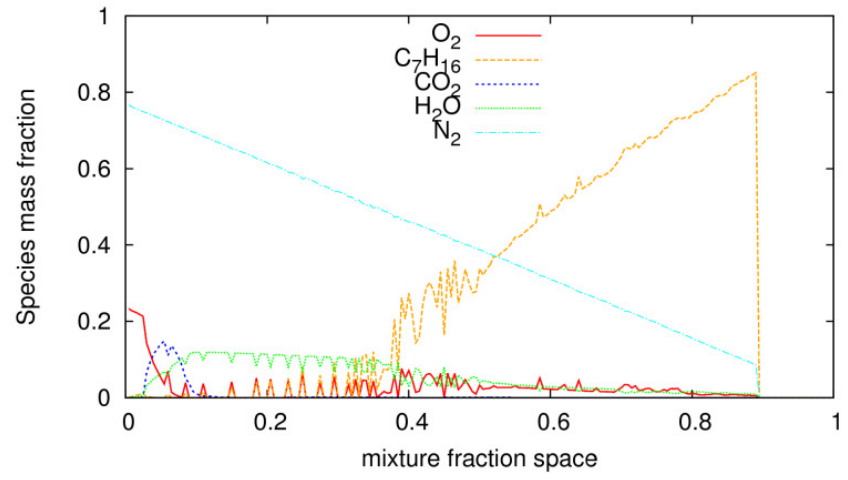

Figure 12: Mass fractions of individual chemical species within the mixture fraction space of the LEM at $t=1.1 \mathrm{~ms}$

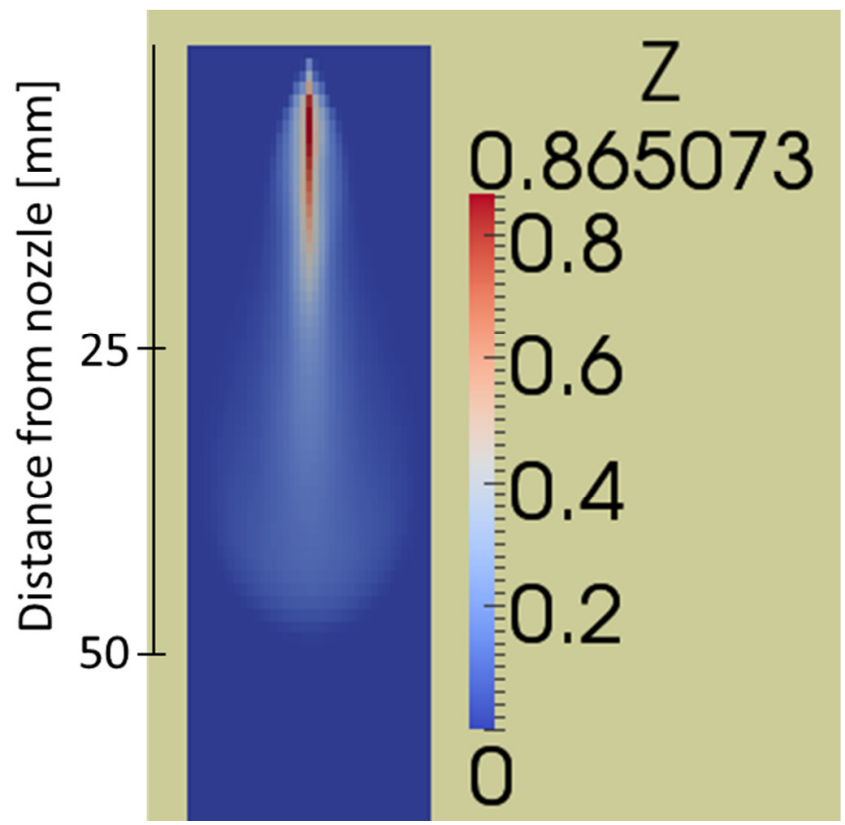

Figure 13: Mixture fraction distribution within the CFD domain at $t=1.1 \mathrm{~ms}$. The figure shows data for a plane extending through the middle of the chamber.

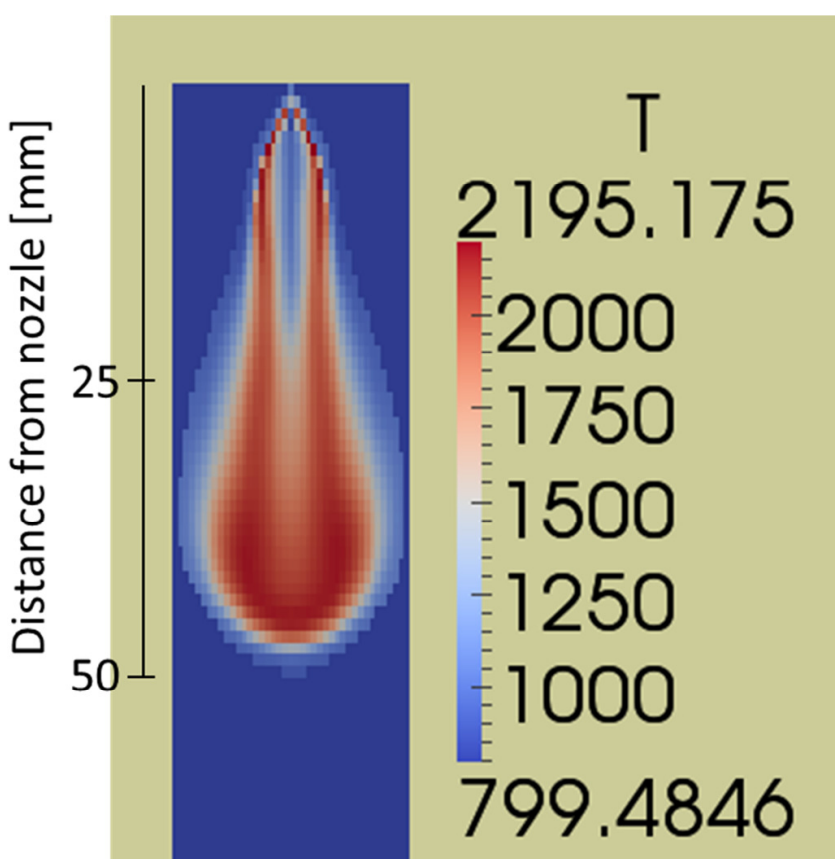

Figure 14: Temperature distribution within the CFD domain at $t$ $=1.1 \mathrm{~ms}$. The figure shows data for a plane extending through the middle of the chamber.

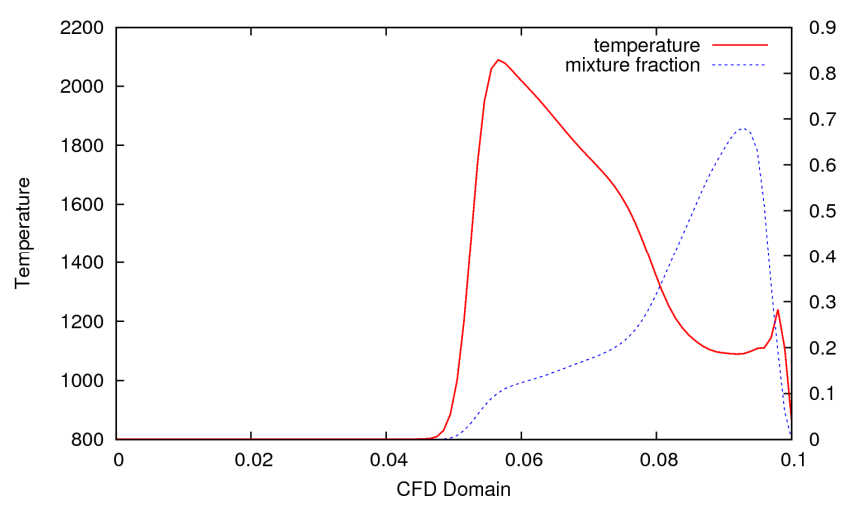

Figure 15: Interpolated values of the temperature and mixture fraction on a line running along the length of the simulated combustion chamber (in the y direction as defined in Figure 3) within the CFD domain at $t=1.1 \mathrm{~ms}$. On the horizontal axis of the plot, 0 corresponds to a position at the bottom of the chamber and a value of 0.1 corresponds to a position immediately below the nozzle.

\section{Summary/Conclusions}

A new model for turbulent non-premixed combustion based on the linear eddy model has been developed. Regimeindependent combustion modeling is achieved by using a representative linear eddy model (LEM), which enables 
regime-independence because it accounts for the local impact of the turbulent motion of the flow on the chemistry of combustion. The representative LEM is solved concurrently with the advancement of the CFD simulation and ensures a direct interaction between the evolving flow solution on the CFD side and the combustion process that can be resolved at all length and time scales along the one-dimensional LEM line. As a first qualitative test the new model was used to simulate a high pressure, high temperature spray combustion process involving $\mathrm{n}$-heptane. Promisingly, the predicted ignition delay times and temperatures are in reasonable agreement with previous results. Unsurprisingly, given that RILEM is a new model, it has several issues that remain to be addressed. In particular, it will be necessary to study the model's statistical properties such as the variation in the scalar dissipation rate and the consistency of the solutions obtained on the LEM and CFD sides. In addition, many improvements of the model can be envisaged, some of which could be realized by using techniques that have been fruitful with flamelet approaches. For example, as was the case in RIF models, it should be straightforward to use multiple representative LEMs. In addition, the model will be validated by comparing its output to that of existing models and experimental data.

\section{References}

1. A.R. Kerstein: One-dimensional turbulence: model formulation and application to homogeneous turbulence, shear flows, and buoyant stratified flows. J. Fluid Mech. 392, 277-334, 1999.

2. D . O. Lignell, A. R. Kerstein, G. Sun, E. I. Monson: Mesh adaption for efficient multiscale implementation of onedimensional turbulence, Theor. Comput. Fluid Dyn., Vol. 27, Issue 3-4, pp 273-295, 2013.

3. Kosters, A., Golovitchev, V., Karlsson, A., "Numerical study of the effect of EGR on flame lift-off in n-heptane sprays using a novel PaSR model implemented in OpenFOAM,"SAE Int.J.Fuel and Lubricants 5(2), 2012.

4. T. M. Smith, S. Menon: One-dimensional simulations of freely propagating turbulent premixed flames, Combustion Science and Technology, 128, 99-130, 1997

5. D. Goodwin: Cantera: An object-oriented software toolkit for chemical kinetics, thermodynamics, and transport processes. http://code/google.com/p/cantera

6. F. Maroteaux, L. Noel: Development of a reduced nheptane oxidation mechanism for $\mathrm{HCCl}$ combustion modeling. Combustion and Flame, 146, 246-267, 2006.

7. Echekki, T., "Turbulent Combustion Modeling," chapter The emerging Role of Multiscale Methods in Turbulent Combustion, pages 177-192. Springer, 2011.
8. El-Asrag, H., and Menon, S. "Large eddy simulation of soot formation in a turbulent non-premixed jet flame," Combustion and Flame, 156:385-395,2009.

9. El-Asrag, H., Lu, T., Law, C. K., and Menon, S., "Simulation of soot formation in turbulent non-premixed flames", Combustion and Flame, 150:108-126, 2007.

10. Kerstein, A. R., "Linear eddy modeling of turbulent transport and mixing," Combustion Science and Technology, 60:391-421, 1988.

11. Kerstein, A.R., "Linear-eddy modeling of turbulent transport. II: Application to shear layer mixing," Combustion and Flame, 75:397-413, 1989.

12. Kerstein, A.R., "Linear-eddy modeling of turbulent transport. Part 6. Microstructure of diffusive scalar mixing," Journal of Fluid dynamics, 240:289-313, 1992.

13. Kerstein, A.R., "Linear-eddy modeling of turbulent transport. Part 7. Finite-rate chemistry and multi-stream mixing," Journal of Fluid Mechanics, 240:289-313, 1992.

14. Kerstein, A.R., "Linear-eddy modeling of turbulent transport. Part 4. Structure of diffusion flames," Combustion Science and Technology, 81:75-96, 1992.

15. Klein, R., "Numerics in Combustion," In L. Vervisch D. Veynante, editor, Introduction to Turbulent Combustion, Brussels, Belgium, January 6-9, 1999. Von Karman Institute for Fluid Dynamics.

16. Meyer, D. W., and Jenny, P., "A mixing model for turbulent flows based on parametrized scalar profiles," Physics of Fluids, 18:035105, 2006.

17. Oevermann, M., Schmidt, H., and Kerstein A. R., "Linear eddy modeling of auto ignition under $\mathrm{HCCl}$ conditions," Combustion and Flame, 155:370-379, 2008.

18. Patel, N., and Menon S., "Simulation of spray-turbulenceflame interactions in a lean direct injection," Combustion and Flame, 155:228-257, 2008.

19. Pitsch H., Chen, M., and Peters N., "Unsteady flamelet modeling of turbulent hydrogen-air diffusion flames," Proceedings of the Combustion Institute, p. 1057-1064, 1998.

20. Sankaran V., and Menon S. "Subgrid combustion modeling of 3-d premixed flames in the thin-reaction-zone regime," In Proceedings of the Combustion Institute, 30:575-582, 2005.

21. Sen B. A., "Linear eddy mixing based tabulation and artificial neural networks for large eddy simulation of turbulent flames," Combustion and Flame, 157:62-74, 2010.

22. Sen B. A., and Menon S., "Large eddy simulation of extinction and reignition with artificial neural networks 
based chemical kinetics," Combustion and Flame, 157:566-578, 2010.

23. Some, K., and Menon S., "Effect of subgrid modeling on the in-cylinder unsteady mixing process in a direct injection engine," Journal of Engineering for Gas Turbines and Power, 125:435-443, 2003

24. Steeper R., Sankaran V., and Oefelein J., "Simulation of the effect of spatial fuel distribution using a linear-eddy model," SAE Technical Paper Series, 2007-01-4131, 2007.

25. Pitsch, H., Wan, Y. P., and Peters N., "Numerical Investigation of Soot Formation and Oxidation Under Diesel Engine Conditions," SAE Technical Paper 952357, 1995.

26. Wakuri, Y., Fujii, M. Amitani, T., Tsuneya R., "Studies on the Penetration of Fuel Spray in a Diesel Engine," Bulletin of J.S.M.E., Vol.3, No. 9, 1960.

27. Ciezki, H. K., Adomeit, G., "Shock tube investigation of Self-Ignition of $n$-heptane-air Mixtures under engine relevant Conditions," Combustion and Flame, 93: 421-433, 1993.

\section{Acknowledgments}

The authors kindly acknowledge the financial support of Chalmers Combustion Engine Research Center (CERC) 